THE ASTROPHYSICAL JouRnAL, 450:280-288, 1995 September 1

(C) 1995. The American Astronomical Society. All rights reserved. Printed in U.S.A.

\title{
EXACT SOLUTIONS FOR STEADY STATE INCOMPRESSIBLE MAGNETIC RECONNECTION
}

\author{
I. J. D. Craig and S. M. Henton \\ University of Waikato, Hamilton, New Zealand \\ Received 1994 November 8; accepted 1995 March 13
}

\begin{abstract}
The problem of steady state magnetic reconnection in incompressible, resistive plasmas is addressed. It is shown that families of exact analytic solutions can be derived by exploiting the formal symmetry between the magnetic field lines and the streamlines of the flow. These solutions, while incorporating previous models for antiparallel magnetic merging, assume no inherent topological symmetry for the reconnective geometry. It is demonstrated that fast reconnection occurs within a narrow $X$-type configuration defined by the intersection of two separatrix lines. One separatrix line is undistinguished physically, but the other is contiguous with a global current sheet across which there is no plasma flow. The implication is that fast reconnection is more likely to be associated with strong shearing motions close to the neutral point rather than with the conventional stagnation point flow topology.
\end{abstract}

Subject headings: MHD - plasmas

\section{INTRODUCTION}

Magnetic annihilation plays a fundamental role in the theory of astrophysical plasmas. In particular, magnetic reconnection - a resistive process involving the cutting and rejoining of field lines - is the only mechanism allowing topological simplification of the global magnetic field (Forbes \& Priest 1987; Biskamp 1994). More contentious, in view of the low resistivities of astrophysical plasmas, is the rate of the magnetic collapse, that is, the rate at which magnetic energy can be transferred to the fluid either as ohmic heating or the kinetic energy of mass motion. It is not clear, for example, that any resistive mechanism is fast enough to explain the explosive phase of the solar flare.

Mechanisms for "fast" magnetic reconnection have been extensively investigated over the last 30 years but an exact analysis, valid for arbitrary plasma resistivities, has proved elusive. So far, exact models have been derived only for the annihilation of strictly antiparallel magnetic fields under the assumption of stagnation point flow (see e.g., Gratton et al. 1988; Phan \& Sonnerup 1990; Jardine et al. 1992). In this paper we present some exact analytic solutions for steady state incompressible, magnetic reconnection. For simplicity, we concentrate on planar geometries but emphasize that our solutions can be readily extended to three-dimensional topologies.

In $\S 2$ we outline the properties of the reconnective system and summarize the impact of previous analytic approaches-a detailed review is provided in the Appendix. Section 3 provides a systematic treatment of the problem of antiparallel magnetic merging. Our central results are presented in $\S 4$, where we construct exact solutions for the reconnective merging of twodimensional magnetic fields.

\section{STEADY STATE RECONNECTION ANALYSIS}

\subsection{Steady State Equations}

The equations governing the reconnective system are the steady state momentum and induction equations for an incompressible plasma in a planar geometry. We introduce nondimensional variables and express fluid velocities in units of the Alfvén speed at the boundary of the region (say $0 \leq|x|$, $|y| \leq 1)$. The system is then described by

$$
\begin{gathered}
(v \cdot \nabla) v=-\nabla^{2} \psi \nabla \psi-\nabla p, \\
E+v \cdot \nabla \psi=\eta \nabla^{2} \psi,
\end{gathered}
$$

where $\psi$ denotes a flux function for the magnetic field $\boldsymbol{B}=\boldsymbol{\nabla}$ $x \psi \hat{z}$ and $p$ is the plasma pressure.

The problem is specified by two parameters $\eta$ and $E$. Here the nondimensionalized resistivity $\eta$ defines the inverse magnetic Reynolds number of the system. Since classical resistivities are extremely small, typically of order $10^{-10}$, rapid magnetic dissipation can only occur in localized regions of high current. In steady state, the resistive annihilation of the field is balanced by the advection of "external " magnetic flux into the diffusion region. In particular, the parameter $E$ defines the magnitude of a uniform electric field driving an external plasma flow. Historically, "fast" reconnection theory has been concerned with demonstrating that, for well-defined external conditions, advective flows can be constructed in which $E$ is effectively independent of the plasma resistivity.

To satisfy the incompressibility condition we introduce a steam function $\phi$ for the velocity field: $v=\nabla \times \phi \hat{z}$. The advection of the magnetic field, $v \cdot \nabla \psi$, is then represented by the Poisson bracket

$$
[\psi, \phi]=\psi_{x} \phi_{y}-\psi_{y} \phi_{x}
$$

where we use subscript notation to indicate differentiation of the dependent variables. We now eliminate the plasma pressure by taking the curl of the momentum equation to obtain

$$
\begin{gathered}
{\left[\nabla^{2} \phi, \phi\right]=\left[\nabla^{2} \psi, \psi\right],} \\
E+[\psi, \phi]=\eta \nabla^{2} \psi .
\end{gathered}
$$

The system is clearly invariant to rotations and translations. The equations are nonlinear, but the symmetry in $\phi$ and $\psi$ suggests that analytic solutions may be possible in certain cases. We first make some preliminary observations.

\subsection{Properties of the Resistive System}

We begin by considering solutions in which certain Poisson brackets vanish. The obvious solution $\phi=\psi$, valid for 
$E=\eta=0$, corresponds to $[\psi, \phi]=0$, but more generally

$$
[\psi, \phi]=0 \Leftrightarrow \psi=f(\phi),
$$

where $f$ is some arbitrary function. Thus, $\psi$ remains constant on the level surfaces of $\phi$.

Suppose, for example, that the momentum brackets in equation (2.4) vanish. In general we must have

$$
\nabla^{2} \phi=-\omega(\phi), \quad \nabla^{2} \psi=-J(\psi),
$$

so the current density $J=J \hat{z}$ is uniform on any magnetic field line while the vorticity $\omega=\omega \hat{z}$ is constant on any streamline.

The momentum brackets also vanish if any dependent variable is a function of only one coordinate-say $\psi=\psi(x)$ - or if any variable takes the harmonic form

$$
H(z)=\sum_{m} a_{m} \frac{z^{m}}{m},
$$

where $z=r e^{i \theta}=x+i y$. It follows that the momentum brackets vanish for a one-dimensional field advected by the harmonic stream function $\phi=\alpha x y=\alpha \operatorname{Im}\left(z^{2} / 2\right)$. This major simplification occurs in the problem of antiparallel magnetic merging under the Ansatz of stagnation point flow (Parker 1963; Sonnerup \& Priest 1975).

Turning to the induction equation, we note that solutions in which the advection term vanishes, that is, $\psi=f(\phi)$, are of little interest to the reconnection problem. However, it is worth remarking that

$$
\phi=\alpha H(z), \quad \psi=\beta H(z),
$$

with $\alpha$ and $\beta$ constant, provides an exact solution for $E=0$ and $\eta$ arbitrary. This solution describes a current-free equilibrium with fluid motions along the field lines maintained by gradients in the plasma pressure.

\subsection{Reconnection Symmetries}

For the traditional reconnection symmetries, viz.,

$$
\phi_{y}=\psi_{x}=0 \text { on } x=0, \phi_{x}=\psi_{y}=0 \text { on } y=0 \text {, }
$$

it is sufficient to model just one quadrant of the reconnection region. The flux and stream functions are even and odd functions, respectively, and the lowest order contribution to the velocity field is the stagnation point flow solution $\phi=\alpha x y$.

The traditional symmetries have an interesting implication: if low-order expansions for $\phi$ and $\psi$ are assumed close to the neutral point, the magnetic field lines osculate-become locally one-dimensional - rather than meet at a finite angle (Priest \& Cowley 1975; Biskamp 1994). This reflects the tendency of the field to form sheets as opposed to localized strands of current. We shall find that this tendency is maintained when the traditional reconnection symmetries are relaxed (see $\S 4$ ).

\subsection{Analytic Modeling}

Virtually all analytic studies of magnetic merging assume the reconnection symmetries of equation (2.10). The impact of kinematic modeling, self-similar analysis, and sub-Alfvénic expansion methods is described in the Appendix. For the moment, we note that most analytic approaches share a common limitation, namely, the assumption that the momentum brackets vanish or are negligible in some sense. The impact of these and other simplifications - for instance, the assumption of a localized diffusion region-makes it difficult to construct an unambiguous theoretical model. Accordingly, in the following analysis, we concentrate on developing exact solutions to the magnetic reconnection problem. Our ultimate goal is to construct models in which none of the Poisson brackets vanish - for then no special symmetries or conservation properties are imposed a priori.

\section{ANTIPARALLEL MAGNETIC MERGING}

\subsection{The Solution}

We now consider the merging of a strictly one-dimensional magnetic field but make no prior assumption as to the nature of the flow. In accordance with $\S 2.2$, we have that

$$
\psi=\psi(x) \Rightarrow\left[\nabla^{2} \phi, \phi\right]=0 .
$$

Since the advection bracket $[\psi, \phi]=\psi_{x} \phi_{y}$ can only depend upon $x$ (by eq. [2.5]), we must take

$$
\phi=y u(x)+q(x),
$$

but to satisfy the momentum bracket (eq. [3.1]) requires

$$
u u^{\prime \prime \prime}=u^{\prime} u^{\prime \prime}, \quad u q^{\prime \prime \prime}=q^{\prime} u^{\prime \prime} \text {. }
$$

Clearly, any solution $u(x)$ satisfies $u^{\prime \prime}= \pm k^{2} u$, where $k$ is some constant. It follows that

$$
u=\alpha x, \quad u=\alpha \sin (k x), \quad u=\alpha \sinh (k x),
$$

with $\alpha$ constant, are the only admissible solutions which vanish on $x=0$. Contributions from $q(x)$ can be discarded if we demand, according to the traditional symmetries $(\S 2.4)$, that no plasma crosses the $x$-axis; otherwise, we take

$$
q=\frac{1}{2} \gamma x^{2}, \quad q=\gamma \cos (k x), \quad q=\gamma \cosh (k x),
$$

with $\gamma$ constant, as solutions corresponding to the respective members of equation (3.4).

It remains only to integrate the induction equation in the form

$$
E+u(x) \psi^{\prime}=\eta \psi^{\prime \prime} .
$$

Introducing the functions

$$
U^{ \pm}(x)=\exp \pm \int_{0}^{x} u \frac{d x}{\eta},
$$

and noting that $U^{-}(x)$ provides an integrating factor for equation (3.6), we express the solution in the form of a quadrature

$$
\psi(x)=\frac{E}{\eta} \int_{0}^{x} d s U^{+}(s) \int_{0}^{s} d t U^{-}(t) .
$$

In the case $u=\alpha x$, the solution is conveniently expressed in terms of the Dawson integral function introduced in $\S 4.2$.

\subsection{Physical Interpretation}

The previous analysis provides a generalization of the wellknown stagnation point flow model which assumes $\phi=\alpha x y$ a priori (Parker 1963; Sonnerup \& Priest 1975). The only new features are the slightly more general inflow profiles (eq. [3.4]) and the possibility of shearing flows for nonvanishing $q(x)$ in equation (3.5). Solutions for the magnetic field and plasma flow profiles are shown in Figure 1a. Contours of a typical sheared stream function are displayed in Figure $1 b$ for the case $q(x)=\gamma$ $\cos (\pi x / 2)$.

Close to the origin all solutions (eq. [3.4]) reduce to stagnation point flow: $\phi=\alpha k x y$. Expanding $\psi$ for small $x$ gives

$$
\psi(x)=\frac{E}{\eta} \frac{x^{2}}{2}\left(1+\frac{\alpha k}{\eta} \frac{x^{2}}{6}+\cdots\right) .
$$




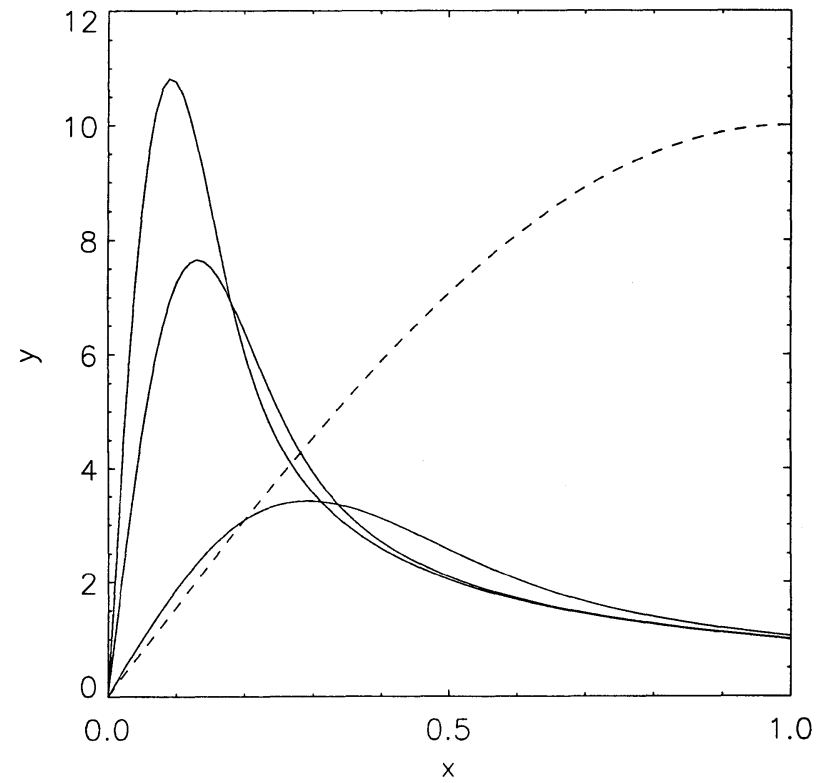

Fig. $1 a$

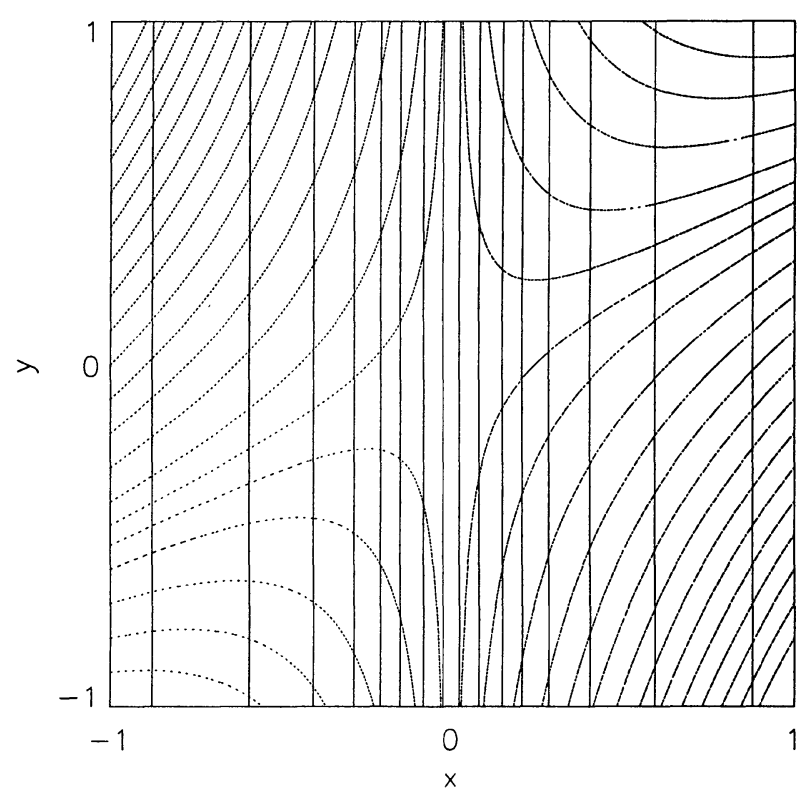

FIG. $1 b$

FIG. 1. (a) Magnetic field (solid lines) and normalized plasma inflow (dashed line) for $E=1, \eta=0.05,0.01$, and 0.005 . The buildup of flux at the onset of the current sheet is most apparent for the smaller values of the plasma resistivity. (b) Contour plots of the streamlines (dotted lines) and the magnetic field (solid lines) for the parameters $E=1, \eta=0.01, \alpha=-1, \beta=0, \gamma=1$. An inflow of the form $\phi=\alpha y \sin (\pi x / 2)$ is assumed.

Thus, if $E$ can be chosen independently of $\eta$, then $x \sim \eta^{1 / 2}$ is the length scale associated with the magnetic field variation close to the neutral line.

We are interested in whether the flux annihilation is "fast." Therefore, we choose $E, \alpha$, and $k$ to be fixed numbers of order unity - the shear parameter $\gamma$ has no influence on the scalingand consider the effect of systematically reducing $\eta$. Then the magnetic field intensity and flow speed on the boundary $(x=1)$ remain unaltered but, as Figure $1 a$ indicates, the field at the onset of the diffusion layer builds up as $\eta^{-1 / 2}$ (see $\S 4.2$ for a formal proof). This flux pileup conspires with the decreasing width of the current layer $\left(x \sim \eta^{1 / 2}\right)$ to maintain the invariance of the flux annihilation rate $E=-\eta J$ at the neutral line. However, as the simplest dimensional arguments confirm, the current in the sheet and the ohmic dissipation rate both increase with the ultrafast scaling $\eta^{-1 / 2}$. Obviously, it is the buildup in the magnetic field intensity at the onset of the sheet that causes the radical departure from the "slow" SweetParker scalings.

\subsection{Summary}

We have shown that antiparallel merging naturally implies sheared, stagnation point flow solutions. This contrasts with previous approaches in which "fast" annihilation models are constructed by taking some Ansatz for the fluid velocity field (see references in $\S 1$ ). A recent example is provided by Besser, Bienat, \& Rijnbeek $(1990,1991)$ who assume stream functions of the form of equation (3.2) and demonstrate magnetic annihilation in plasmas of finite velocity.

More generally, as discussed in the Appendix, it appears that magnetic reconnection can only be achieved by considering - departures from local stagnation point flow. Even so, under the standard symmetries $(\S 2.3)$, reconnection seems limited to osculating rather than $X$-type field geometries.

\section{MAGNETIC RECONNECTION SOLUTIONS}

\subsection{The Solution}

We recall from $\S 2.2$ that the momentum brackets vanish in the case of one-dimensional and harmonic variables. However, if we take

$$
\phi=f(x)+F(x, y), \quad \psi=g(x)+G(x, y),
$$

where $F$ and $G$ are harmonic, there are nonlinear contributions which prevent the momentum brackets from vanishing. If we specialize to the case of equation (2.9),

$$
F=\alpha H(x, y), \quad G=\beta H(x, y),
$$

where $\alpha$ and $\beta$ are constant, then an equilibrium exists (for $f=g=E=0$ ) in which irrotational flows are constrained to potential field lines. Also, by taking $\beta=0$, we can expect to recover the harmonic annihilation solution of $\S 3$.

Assuming equations (4.1) and (4.2), the momentum brackets reduce to

$$
\left(\alpha f^{\prime \prime \prime}-\beta g^{\prime \prime \prime}\right) H_{y}=0 .
$$

This implies

$$
f(x)=\frac{\beta}{\alpha} g(x)+q(x),
$$

where $q$ is some arbitrary quadratic function. Since the global superposition of a uniform velocity field has no inherent interest, we choose $q(x)=\frac{1}{2} \gamma x^{2}$.

The advection bracket yields

$$
[\psi, \phi]=\left(\alpha g^{\prime}-\beta f^{\prime}\right) H_{y},
$$

but since the current is one-dimensional, we must choose $H_{y}$ to eliminate any $y$-dependency. Since $H$ is harmonic, $H=x y$ is the only admissible solution. 
The induction equation now reduces to

$$
E-\beta \gamma x^{2}+h(x) g^{\prime}=\eta g^{\prime \prime},
$$

where

$$
h(x)=\left(\frac{\alpha^{2}-\beta^{2}}{\alpha}\right) x .
$$

The solution can be expressed in the quadrature form

$$
g(x)=\frac{E}{\eta} \int_{0}^{x} d s U^{+}(s) \int_{0}^{s} d t\left(1-\frac{\beta \gamma}{E} t^{2}\right) U^{-}(t),
$$

where $U^{ \pm}$is given by equation (3.7) on replacing $u(x)$ by $h(x)$.

$$
\text { 4.2. The Solution for } \gamma=0
$$

The general solution, viz.,

$$
\phi=\alpha x y+\frac{1}{2} \gamma x^{2}+\frac{\beta}{\alpha} g(x), \quad \psi=\beta x y+g(x),
$$

is specified by three internal parameters $\alpha, \beta$, and $\gamma$. The parameter of least significance is $\gamma$ : its role, as in the antiparallel merging problem, is simply to superpose an admissible shearing flow on the preexisting velocity field. Mathematically, this is accomplished by adding a particular integral to the solution of the homogeneous equation for $g(x)$.

An explicit form for the homogeneous solution can be obtained using the Dawson integral (Spanier \& Oldman 1987)

$$
\begin{aligned}
\operatorname{daw}(x) & =\int_{0}^{x} \exp \left(t^{2}-x^{2}\right) d t \\
& =\frac{\operatorname{sgn}(x)}{2} \int_{0}^{x^{2}} \exp \left(t-x^{2}\right) \frac{d t}{\sqrt{t}} .
\end{aligned}
$$

This function increases as $x-2 x^{3} / 3$ for small $x$; it peaks when daw $(x) \simeq 0.541$ at the point $x=x_{m} \simeq 0.924$, before declining monotonically, as $1 /(2 x)$ for large $x$.

Equation (4.7) with $\gamma=0$ implies that

$$
g^{\prime}(x)=\frac{E}{\eta \mu} \operatorname{daw}(\mu x), \quad \mu^{2}=\frac{\left(\beta^{2}-\alpha^{2}\right)}{2 \alpha \eta} .
$$

The current density takes the form

$$
g^{\prime \prime}(x)=\frac{E}{\eta}[1-2 \mu x \text { daw }(\mu x)] .
$$

and the magnetic and velocity fields are given by

$$
\begin{aligned}
& \boldsymbol{B}=\beta x \hat{\boldsymbol{x}}-\left[\beta y+\frac{E}{\eta \mu} \operatorname{daw}(\mu x)\right] \hat{\boldsymbol{y}}, \\
& \boldsymbol{v}=\alpha x \hat{\boldsymbol{x}}-\left[\alpha y+\frac{\beta}{\alpha} \frac{E}{\eta \mu} \operatorname{daw}(\mu x)\right] \hat{\boldsymbol{y}} .
\end{aligned}
$$

Figure 2 shows a plot of daw $(\mu x)$ and its normalized derivative for $\mu=5$. The physically interesting case of small $\eta$ corresponds to the limit of large $\mu$. In this limit, the magnetic and velocity fields at the boundary $x=1$ become independent of $\eta$, as required for fast reconnection: specifically, $g^{\prime}(x) \rightarrow-E$ / $h(x)=\alpha E /\left[\left(\beta^{2}-\alpha^{2}\right) x\right]$ as $x \rightarrow 1$. This implies that the integrated sum of the reverse currents in the region $\mu x>1$-see the derivative plot in Figure 2-almost cancels the total current in the sheet.

The flux and stream functions involve an integral of the Dawson function. Using a hypergeometric function represen-

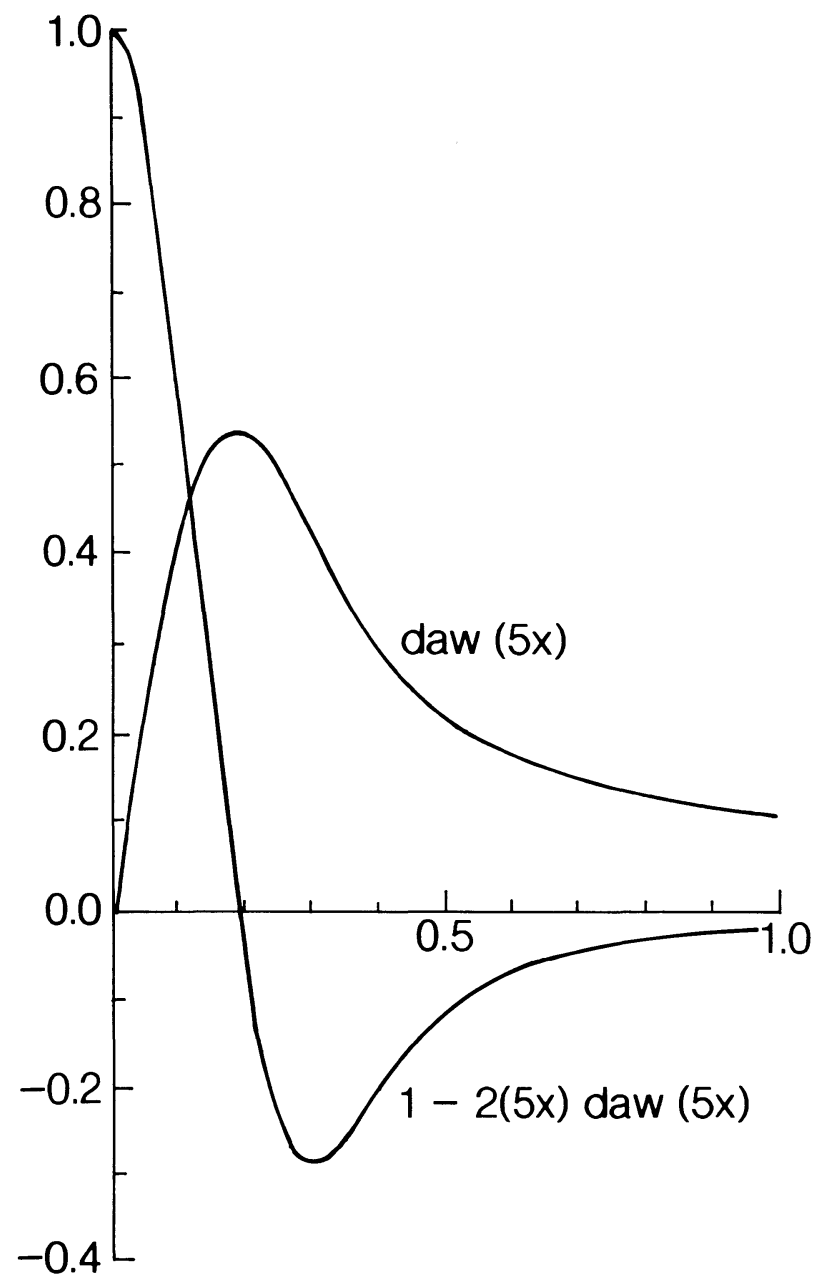

FIG. 2.-Plot of daw $(\mu x)$ vs. $x$ for the case $\mu=5$. Also shown is the current profile (normalized to unity at the origin) given by the derivative function $1-2(\mu x)$ daw $(\mu x)$.

tation (Spanier \& Oldham 1987),

$$
\begin{aligned}
\int_{0}^{x} \operatorname{daw}(t) d t & =\frac{x^{2}}{2} \sum_{i=0}^{\infty} \frac{(1)_{i}}{(3 / 2)_{i}(2)_{i}}\left(-x^{2}\right)^{i} \\
& \equiv \frac{x^{2}}{2}{ }_{1} F_{2}\left(1, \frac{3}{2}, 2,-x^{2}\right),
\end{aligned}
$$

where the Pochhammer symbols are defined by $\left(m_{i}\right)=m(m+$ 1) $(m+2) \ldots(m+i-1)$, we obtain

$$
g(x)=\frac{E}{\eta} \frac{x^{2}}{2}{ }_{1} F_{2}\left(1, \frac{3}{2}, 2,-\mu^{2} x^{2}\right) .
$$

The first few terms of series (4.14) define the behavior at small $x$ :

$$
g(x)=\frac{E}{\eta} \frac{x^{2}}{2}\left(1-\frac{\mu^{2} x^{2}}{3}+\cdots\right),
$$

a result which reduces to the local expansion (3.9) for the case $\beta=0$.

\subsection{Physical Interpretation}

To clarify the interpretation of the solution we fix the external parameters $\eta>0, E>0$, and we assume for the moment that $|\alpha|>|\beta|$ and $\gamma=0$. Then inflow along the $x$-axis corre- 

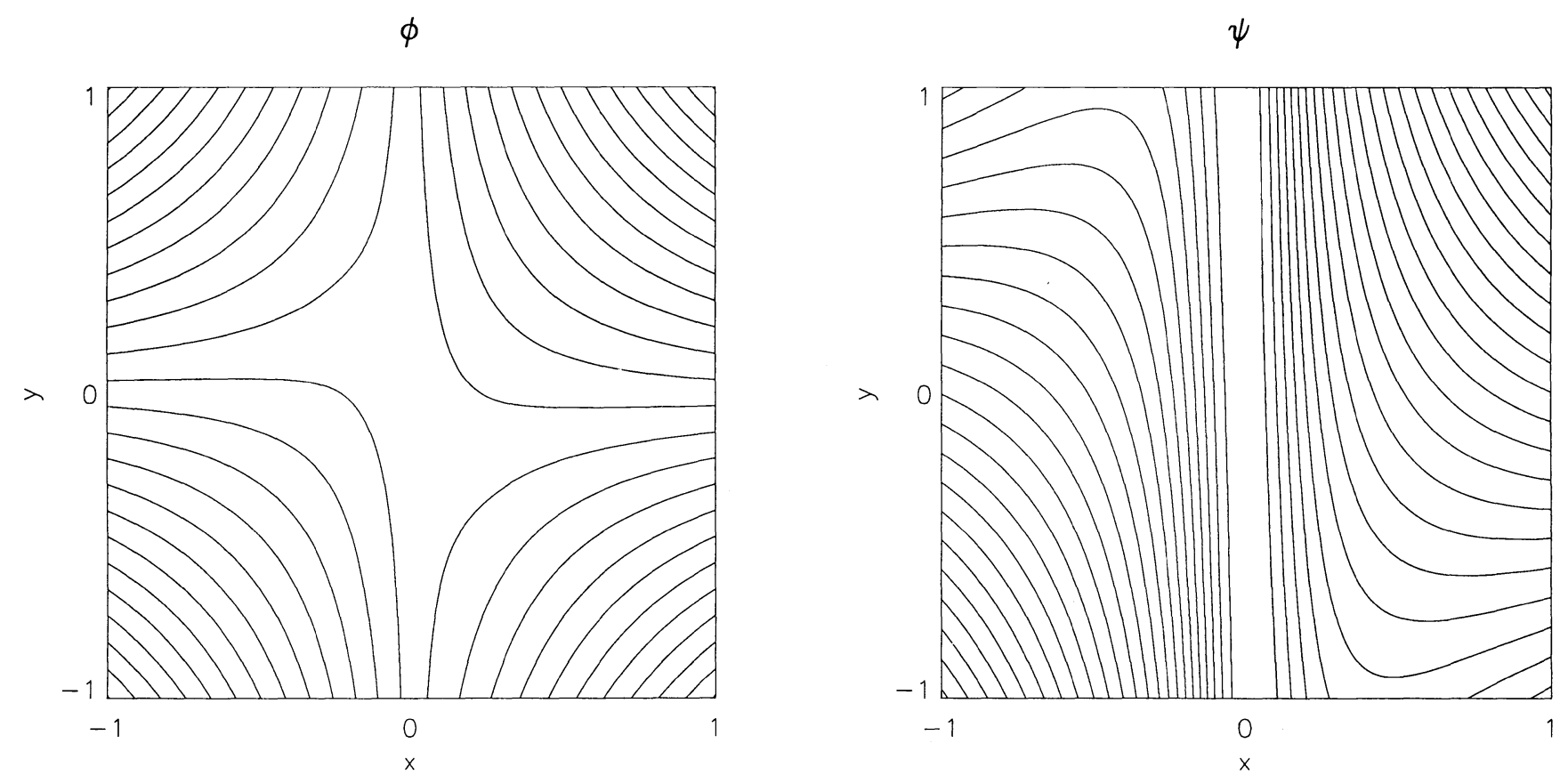

Fig. 3. Streamlines and magnetic field lines of the inflow solution $E=0.1, \eta=0.01, \alpha=-1, \beta=0.25, \gamma=0$

sponds to $\alpha<0$, and the antiparallel merging solution of $\S 3$ is recovered by taking $\beta=0$.

Consider now the behavior of the solution as $\beta$ increases from zero, all other parameters being fixed (with $\alpha<0$ ). As Figure 3 makes clear, the stagnation flow topology is distorted by the development of shear flows close to the origin. (Note that contours of $\phi$ and $\psi$ are selected to emphasize qualitative features of the solution, for instance separatrix lines, rather than to provide accurate measures of the field intensities). For small $\beta$ the antiparallel field becomes weakly two-dimensional, as indicated by the $\psi$ contours, but the current remains aligned to the separatrix $x=0$. As $\beta$ approaches $|\alpha|$, the distortion of the field intensifies and the field lines increasingly resemble the streamlines. When $\beta=|\alpha|$, the advection bracket vanishes and the solution changes character, yielding a uniform current density with $g=f=(E / \eta)\left(x^{2} / 2\right)$. For $\beta>|\alpha|$, the current becomes maximal on the boundaries $|x|=1$ rather than along the $y$-axis. We conclude that $\left(\alpha^{2}-\beta^{2}\right) / \alpha<0$ is necessary to maintain the physically interesting case of localized currents along $x=0$. This also guarantees $\mu^{2}>0$, as required for the Dawson integral representation in equation (4.10).

Now consider the case where $\beta>\alpha>0$. Localized current solutions are again possible, but now there is an outflow of plasma along the $x$-axis. Accordingly, the flux pileup is accomplished mainly by the advection of the $x$-component of field by inflow parallel to the $y$-axis. Increasing $\beta$ has the effect of increasing (decreasing) the shear of the streamlines (field lines). Typical solutions are shown in Figure 4.

\subsection{The Reconnection Region}

The separatrices $\psi=0$ of the magnetic field are defined by the two lines

$$
x=0, \quad y=-\frac{g(x)}{\beta x} .
$$

These lines cut the $x$-axis at the angles $\pi / 2$ and $\tan ^{-1}[-E /$
$(2 \beta \eta)]$, respectively. The intersection of the separatrices defines a narrow wedge of angle $2 \beta \eta / E$ in which reconnected field lines expel plasma in the form of a magnetic slingshot.

The details of the reconnection region are illustrated in the $\phi$ and $\psi$ plots of Figure 5. The local field-line geometry is that of the classical $X$-point but, outside the slingshot wedge, the flow profile is highly sheared, and flow across the curving separatrix line is clearly visible. Yet no flow occurs across the $y$-axis-the separatrix line contiguous with the current sheet.

What determines the penetration of the opposing plasma inflows across the $x$-axis? Clearly, there is a tendency for the flow to shear more severely with increasingly $\beta$. In general, the $y$-component of the fluid velocity vanishes when

$$
y_{m}=-\frac{1}{\alpha}\left[\gamma x+\frac{\beta}{\alpha} g^{\prime}(x)\right] \text {. }
$$

Hence, for $\gamma=0$ and fixed $\beta$ the penetration is governed by the amplitude of the field $g^{\prime}(x)$. The maximum of $g^{\prime}(x)$ occurs when $x \sim \eta^{1 / 2}$ : this effectively defines the shearing length scale for $\beta>0$.

\subsection{The Plasma Pressure}

The pressure is given by

$$
p=p_{0}-\frac{1}{2}\left(u_{H}^{2}+b_{0}^{2}\right)+\beta y b_{0},
$$

where $\boldsymbol{u}_{\boldsymbol{H}}=(\alpha x,-\alpha y), \boldsymbol{b}_{0}=-g^{\prime}(x) \hat{y}$, and $p_{0}$ is constant. The term in $\beta$ accounts for departures from the antiparallel, harmonic merging solution. Pressure contours for the case $\beta=0$ are shown in Figure $6 a$ : presumably the high-pressure region in the center is required to divert the (stagnation point) flow. The plot of Figure $6 b$ confirms that increases in $\beta$ have the effect of skewing the pressure contours.

\section{DISCUSSION AND CONCLUSIONS}

We have discussed analytic solutions for steady state magnetic merging in incompressible plasmas. To our knowledge, 

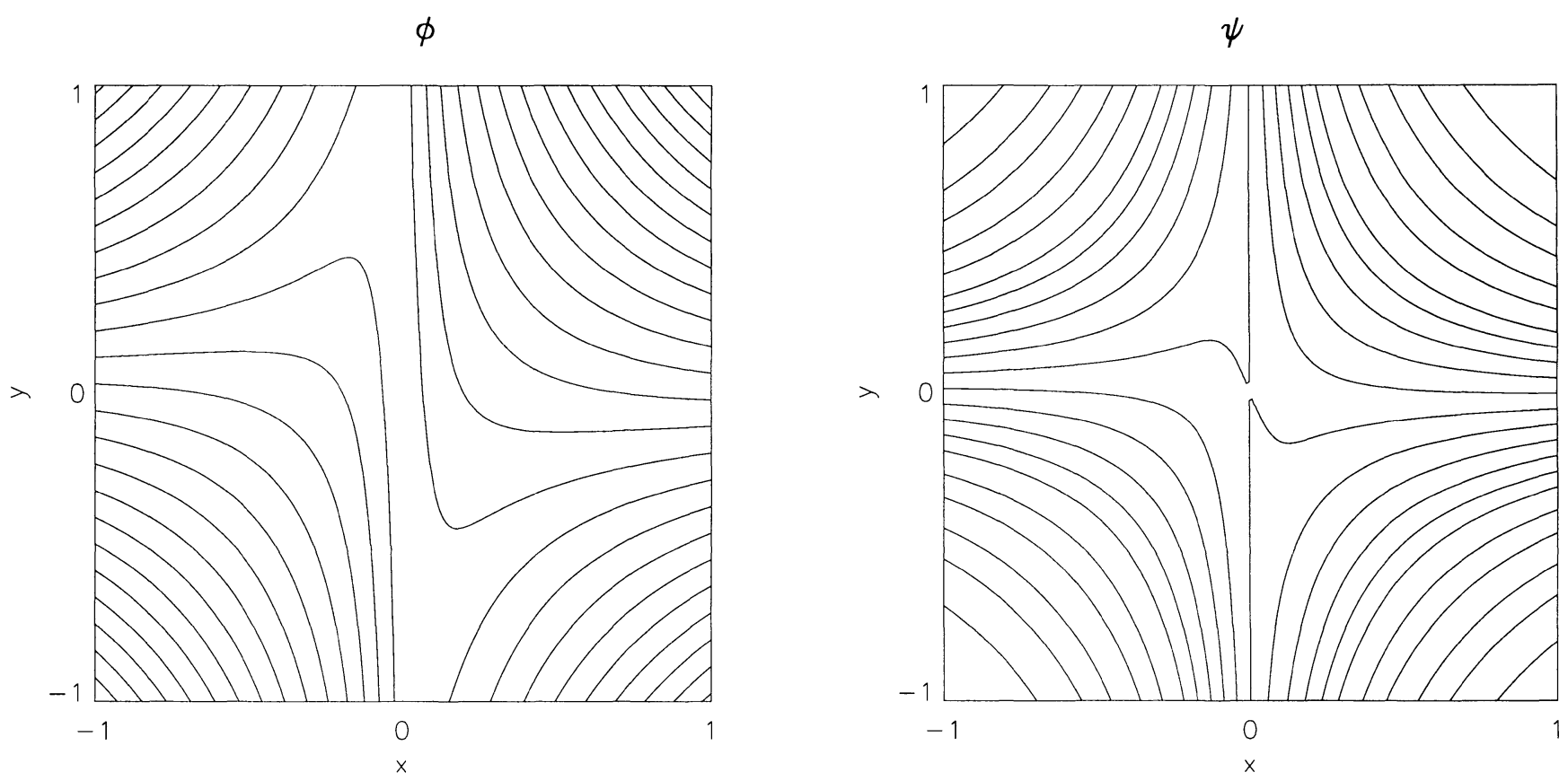

Fig. 4.-Outflow solution of $\phi$ and $\psi$ where $E=0.1, \eta=0.01, \alpha=1, \beta=2, \gamma=0$

the only previous exact solutions are stagnation point flow models (e.g., Parker 1963; Sonnerup \& Priest 1975) which describe the nonreconnective annihilation of antiparallel magnetic fields. We have shown that, in addition to more general annihilation modes, an analytic description is also possible for fast reconnection. The reconnective solutions require no special symmetries or conservation properties for the fluid-in other words, there are no vanishing Poisson brackets in the solution.

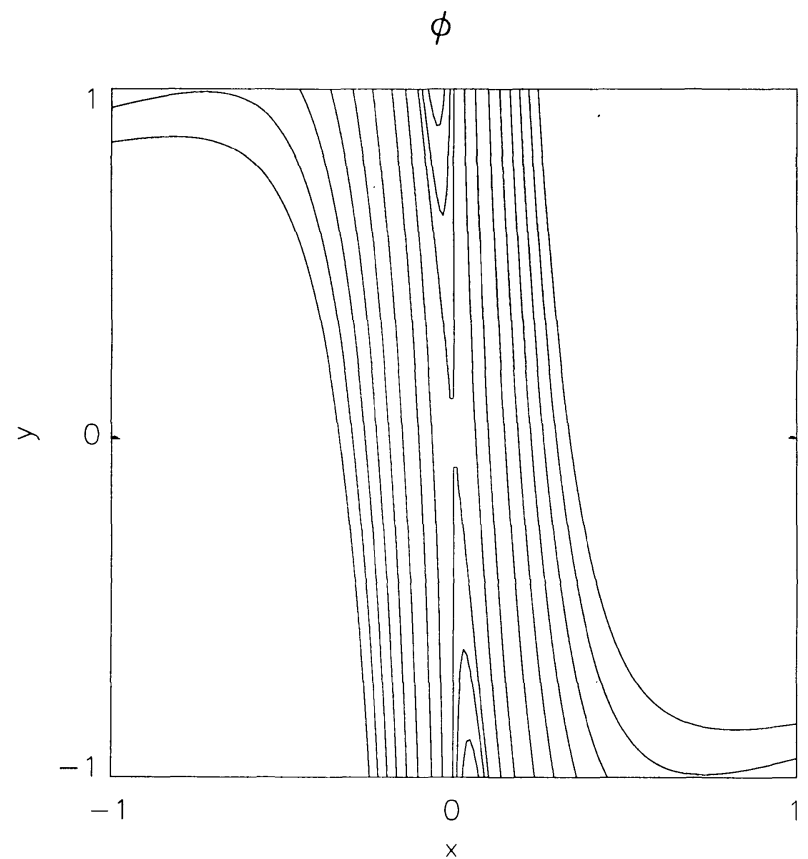

The merging of antiparallel magnetic fields was considered in $\S 3$. All solutions exhibit flux pileup in the vicinity of the neutral line $\left(x \sim \eta^{1 / 2}\right)$, but small length scales are absent in the velocity field. These features are uncompromised by the superposition of admissible shearing flows on the solution. The recent work of Besser et al. $(1990,1991)$ shows that similar merging solutions can also be constructed for viscous fluids.

More significantly, in $\S 4$, we constructed exact solutions for the fast, reconnective annihilation of two-dimensional magnetic

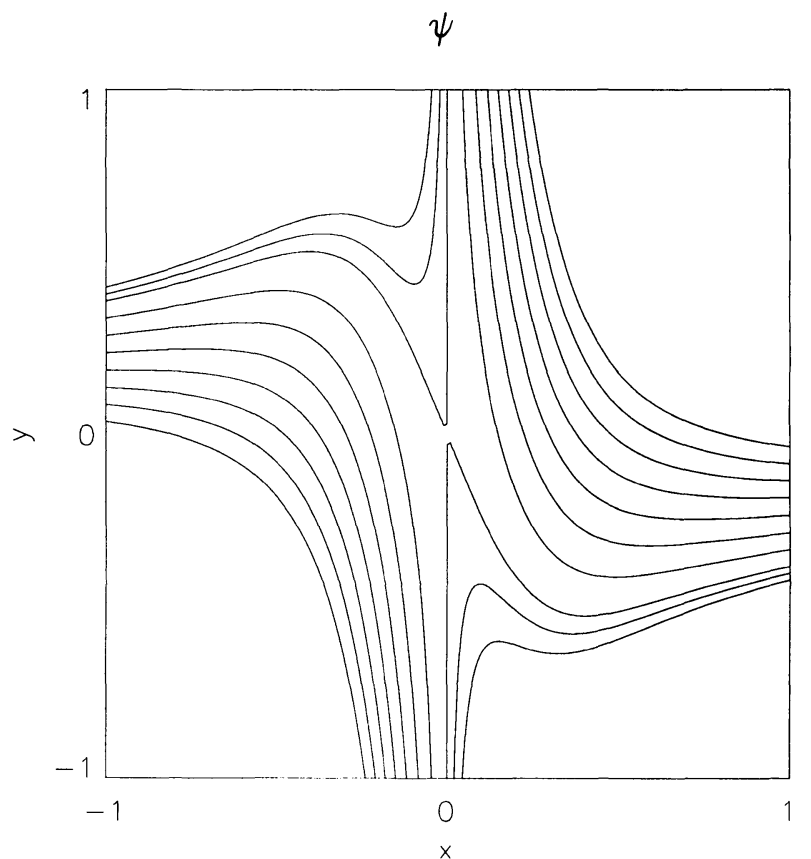

Fig. 5.-Outflow solution for $\phi$ and $\psi$ where $E=1, \eta=0.1, \alpha=1, \beta=2, \gamma=0$. These parameters are chosen to highlight the $X$-point reconnection topology close to the neutral point. Note that the contours have been selected to emphasize the qualitative features of the solution rather than the intensity of the respective fields. 


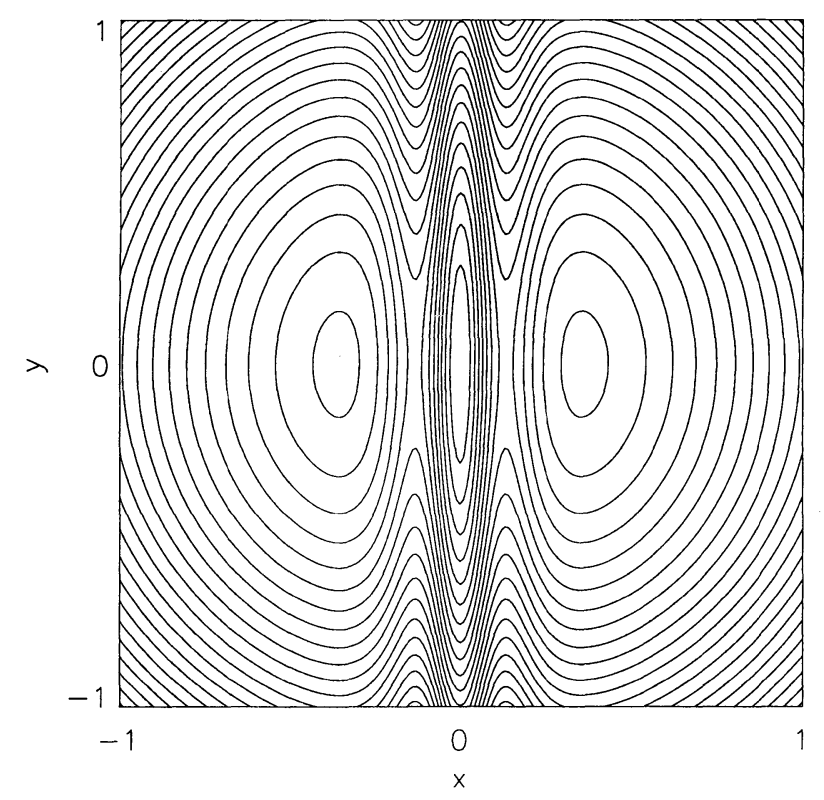

FIG. $6 a$

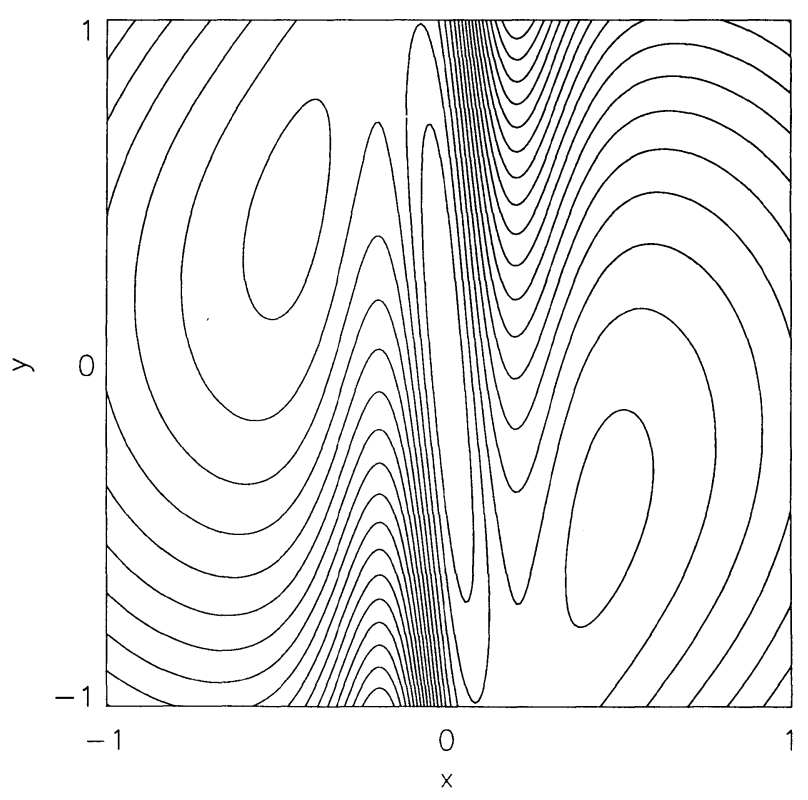

Fig. $6 b$

FIG. 6.-Contours of plasma pressure $p$ for $E=0.1, \eta=0.01, \alpha=-1, \beta=0$ and $0.75, \gamma=0$

fields. Flux pileup is again evident, but departures from strictly one-dimensional merging are associated with relaxing the symmetries required by stagnation point flow (see Forbes $\&$ Priest 1987). Thus, the two-dimensionality of the magnetic field is reflected by the presence of strong shearing motions, aligned to the current layer, close to the neutral point. Fast reconnection occurs via an intense current sheet $\left(J \sim \eta^{-1}\right)$, of width $\sim \eta^{1 / 2}$, which extends to the outer boundary. The thickness of the sheet is reflected in the small length scale of the shear flow. The reconnection region is delineated by the $X$-type intersection of two separatrix lines. Although one separatrix line is undistinguished physically, the other, contiguous with the current sheet, has no flow across it.
On a more general level, we note that many of the features of the reconnection solutions-for instance, the likelihood of extended currents along separatrices - are also nascent under the conventional reconnection symmetries (Craig \& Henton 1994). However, our analysis suggests that fast reconnection is more naturally associated with shear flow topologies than with the more restrictive symmetries required by stagnation point flow.

It is a pleasure to acknowledge helpful discussions with Alfred Sneyd, Ernie Kalnins, Richard Fabling, and Sandy McClymont. We are grateful for the detailed, constructive comments of an anonymous referee.

\section{APPENDIX}

\section{ANALYTIC MODELING OF THE RESISTIVE SYSTEM}

\section{A1. SELF-SIMILAR ANALYSIS}

The analysis of Yeh \& Axford (1970) provides an interesting approach to the reconnection problem. The basic notion is to construct advective region solutions within a cylindrical domain surrounding a "point" dissipation region. Since, in cylindrical coordinates, the brackets in equation (2.3) reduce to

$$
[\psi, \phi]=\psi_{r} \frac{\phi_{\theta}}{r}-\phi_{r} \frac{\psi_{\theta}}{r},
$$

it is natural to seek solutions of the form

$$
\phi=r g(\theta), \quad \psi=r f(\theta) .
$$

In fact, these are the only separable solutions (Yeh \& Axford 1970).

The momentum and induction equations imply that

$$
\begin{gathered}
\left(g^{2}+g g^{\prime \prime}\right)^{\prime}=\left(f^{2}+f f^{\prime \prime}\right)^{\prime} \\
E+f g^{\prime}-g f^{\prime}=0
\end{gathered}
$$


and a particular solution is clearly

$$
g(\theta)=-\frac{E}{A} \sin (\theta+\alpha), \quad f(\theta)=A \cos (\theta+\alpha)
$$

This solution (Sonnerup 1970) is the only nonsingular member of the self-similar family of equation (A1). For $\alpha=0$, the solution reduces to $\phi=-E y / A, \psi=A x$, which represents the uniform advection of a constant magnetic field aligned normal to the flow. More generally, Sonnerup's “ reconnection" model involves patching together several such advective solutions each valid in different sectors of the plane, say $\left(\theta_{1} \leq \theta \leq \theta_{2}\right)$. This patching introduces two radial boundary lines in each quadrant along which the current density and vorticity are nonvanishing (as emphasized by Parker 1973). The MHD equations break down along these lines, and attempts to provide a physical interpretation in terms of slow mode shocks or fluid expansion fans have been unconvincing (see Vasyliunas 1975).

In our opinion, these difficulties are a manifestation of assuming a point current at the origin. Circumstantial evidence is provided by the specter of extended currents along the radial lines $\left(\theta=\theta_{1}, \theta=\theta_{2}\right)$; such discontinuities are consistent with the resistive system being overspecified in some manner (see $\S \mathrm{A} 3$ ).

\section{A2. SUB-ALFVÉNIC SOLUTIONS}

The sub-Alfvénic approach has been systematized by Forbes \& Priest (1987). The essential point is that for slow flow speeds the momentum equation (2.1) is dominated by the Lorentz and pressure forces: formally $\left[\nabla^{2} \phi, \phi\right]$ is second order in the Alfvén Mach number of the plasma and so can be regarded as a perturbation term in the development of sub-Alfvénic solutions. Typically, an expansion is developed only for the advection region solution, the diffusion region being treated approximately as a localized internal boundary of adjustable length. Within these assumptions, families of reconnection models can be constructed, for instance the famous Petschek (1964) model-but see Biskamp $(1986,1994)$ for a dissenting view.

More recently, Craig \& Henton (1994) have explored solutions which retain only the lowest order flow terms in the expansion (see also Craig \& Rickard 1994; Priest et al. 1994). The flux function is then obtained by solving $\nabla^{2} \psi=-J(\psi)$ for specified $J(\psi)$, subject to boundary conditions on $\psi$ and its normal derivative. The key point is that because the induction equation determining the velocity field,

$$
E+[\psi, \phi]=-\eta J(\psi),
$$

can be resolved exactly by the method of characteristics, certain universal features of the problem can be identified. A special role is played by the separatrices of the field: these fan outward from the neutral point and carry intense currents of uniform amplitude to the outer boundary; no flow across the separatrices is permitted, but strong shear flows develop along streamlines adjacent to the separatrices (see also Bulanov, Shasharina, \& Pegararo 1990). Although it is not clear how these features will be modified when the constraint $J=J(\psi)$ is relaxed, we note that all solutions must be dominated by the low-order development, provided that the sub-Alfvénic assumption is not violated.

\section{A3. KINEMATIC SOLUTIONS}

Kinematic solutions have been provided by Parker (1973) under the assumption of stagnation point flow $(\phi=\alpha x y)$. Although much physical insight can be gained from kinematic models, the approach is generally compromised by violation of the momentum equation. We ask: is it possible to develop a self-consistent kinematic approach?

Suppose, for instance, that the prescribed velocity field has the harmonic form $\phi=\alpha x y$. Then the induction equation must be resolved for $\psi$, subject to the consistency condition $J=J(\psi)$.

Using the method of characteristics, equation (A3) implies

$$
\frac{d x}{\alpha x}=-\frac{d y}{\alpha y}=-\frac{d \psi}{E+\eta J(\psi)},
$$

and so the characteristics are just the streamlines $\phi$ (as in Parker 1973).

We introduce the function $I(\psi)$,

$$
I^{-1}(\psi)=-\alpha \int \frac{d \psi}{E+\eta J(\psi)},
$$

and assume that $\psi$ can be arbitrarily specified on the reference line $x=x_{R}$, via $\psi\left(x_{R}, y\right)=\psi_{R}(y)$. Then

$$
I(\psi)=\frac{I\left[\psi_{R}\left(\phi / \alpha x_{R}\right)\right]}{1+I\left[\psi_{R}\left(\phi / \alpha x_{R}\right)\right] \ln \left(x / x_{R}\right)}, \quad \phi=\alpha x y,
$$

provides an implicit expression for $\psi$ in terms of the boundary value data. Clearly, we expect $I(\psi)$ to be well behaved for all analytic current distributions $J(\psi)$ (see Craig \& Henton 1994). But suppose we fix $y$ and let $x \rightarrow 0$ under the assumption that $I\left(\psi_{R}\right)$ remains finite. Then $I(\psi) \rightarrow 0$, but its $x$-derivative becomes unbounded. This can only happen if $\psi_{x}$ or $I^{\prime}(\psi)$ behave badly for small $x$ - possibilities we reject as unphysical!

The implication is that the system is overspecified by the assumption $\phi=\alpha x y$ and the consistency constraint $J=J(\psi)-$ and so the system extracts revenge by developing unphysical singularities. Thus, plausible stagnation point flow solutions can be constructed only for fields which trivially satisfy $J=J(\psi)$, namely, one-dimensional fields. This is certainly consistent with the result that stagnation point flow implies antiparallel merging-and the converse $(\S 3.1)$. 


\section{REFERENCES}

Besser, B. P., Biernat, H. K., \& Rijnbeek, R. P. 1990, Planet. Space Sci., 38, 411 1991, J. Atm. Terr. Phys., 53, 1081

Biskamp, D. 1986, Phys. Fluids, 29, 1520

$$
\text { . 1994, Phys. Rep., 4, } 237
$$

Bulanov, S. V., Shasharina, S. G., \& Pegararo, F. 1990, Plasma Phys. and Controlled Fusion, 32, 5, 377

Craig, I. J. D. \& Henton, S. M. 1994, ApJ, 434, 192

Craig, I. J. D., \& Rickard, G. J. 1994, A\&A, 287, 261

Forbes, T. G., \& Priest, E. R. 1987, Rev. Geophys., 25, 1583

Gratton, F. T., Heyn, M. F., Biernat, J. K., Rijnbeek, R. P., \& Gnavi, G. 1988, J. Geophys. Res., 93, 7318

Jardine, M., Allen, H. R., Grundy, R. E., \& Priest, E. R. 1992, J. Geophys. Res., 97,4199

Parker, E. N. 1963, ApJS, 8, 177
Parker, E. N. 1973, J. Plasma Phys., 9, 49

Petschek, H. E. 1964, in AAS-NASA Symposium on the Physics of Solar Flares, ed. W. N. Hess (NASA SP-50), 425

Phan, T. D., \& Sonnerup, B. U. O. 1990, J. Plasma Phys., 44, 525

Priest, E. R., \& Cowley, S. W. H. 1975, J. Plasma Phys., 14, 271

Priest, E. R., Titov, V. S., Vekstein, G. E., \& Rickard, G. J. 1994, J. Geophys. Res., 99, 21467

Sonnerup, B. U. O. 1970, J. Plasma Phys., 4, 161

Sonnerup, B. U. O., \& Priest, E. R. 1975, J. Plasma Phys., 14, 283

Spanier, J., \& Oldham, K. B. 1987, An Atlas of Functions (New York: Hemisphere)

Vasyliunas, V. M. 1975, Rev. Geophys. Space Phys., 13, 303

Yeh, T., \& Axford, W. I. 1970, J. Plasma Phys., 4, 207 